\title{
Editorial
}

\section{Biomass-Based Materials and Technologies for Energy}

\author{
Jingxin Wang, ${ }^{1}$ Xinfeng Xie, ${ }^{1}$ David B. DeVallance, ${ }^{1}$ Jianchun Jiang, ${ }^{2}$ \\ Lixin Huang, ${ }^{2}$ and Levente Dénes ${ }^{3}$ \\ ${ }^{1}$ Division of Forestry and Natural Resources, West Virginia University, Morgantown, WV 26505, USA \\ ${ }^{2}$ Institute of Chemical Industry of Forest Products, Chinese Academy of Forestry, Nanjing 210042, China \\ ${ }^{3}$ Institute of Product Design and Manufacturing Technologies, University of West Hungary, Bajcsy-Zsilinszky Utca 4, \\ Sopron 9400, Hungary
}

Correspondence should be addressed to Jingxin Wang; jxwang@wvu.edu

Received 17 August 2015; Accepted 17 August 2015

Copyright (C) 2015 Jingxin Wang et al. This is an open access article distributed under the Creative Commons Attribution License, which permits unrestricted use, distribution, and reproduction in any medium, provided the original work is properly cited.

With the development of global industrialization, the world is facing an energy uncertainty and increasing environmental concerns resulting from fossil energy use. Biomass-based materials are known to have lower greenhouse gas emissions than those from nonrenewable resources, such as petroleum and coal. In recent years, biomass-based materials and technologies have become one of the most important components of sustainable systems due to biomass abundance and renewability. Biomass-based materials and technologies have drawn much attention from governments, academia, and industry, having become one of the most active research fields.

This special issue included some of the best contributions from the researchers in the field. Through a thorough and professional process, the best manuscripts were selected for publication in this special issue. The papers in this special issue present the state-of-the-art perspective on the wide spectrum of biomass-based materials and technologies ranging from high value chemicals extraction, materials handling, and chemical conversion to biomass-based composites for various engineering applications.

Understanding and improving the biomass material properties for hot molding processes are critical for effective utilization of biomass for composites. The article by S. Kajikawa and T. Iizuka found that steam or short-time dry heat treatments could improve the fluidity of bamboo particles. The study by L. Peng et al. showed a great potential of wood fiber for manufacturing high quality composites for acoustic applications. The article entitled "Texture Feature
Extraction and Classification of SEM Images of Wheat Straw/Polypropylene Composites in Accelerated Aging Test" enlightens the readers with a novel and effective approach of combining image analysis and artificial intelligence to classify the aging status of biomass fiber/polymer composites.

Biomass is also a cost-effective resource for high value chemicals with pharmacological properties. By combining reduced pressure and ultrasonication, the study by P. Xie et al. made an impressive progress on effectively extracting oleuropein from olive leaves. The method not only reduced the energy consumption for extracting chemicals but also achieved higher yield than traditional methods.

In addition to applications in materials and chemicals, biomass feedstocks play an important role in energy applications. The study by X. Song et al. revealed that larger particle sizes of biomass could be more beneficial in terms of saving milling energy and obtaining higher cellulose recovery and total sugar yield. The article entitled "Microwave Assisted Hydrolysis of Holocellulose Catalyzed with Sulfonated Char Derived from Lignin-Rich Residue" by K. Wang et al. introduced an efficient method (83\% w.t. conversion within 60 minutes) to break the carbohydrate polymer in woody biomass using the lignin-derived char as the catalyst.

\section{Acknowledgments}

We thank the authors for their original contributions and the reviewers for the insightful suggestions which have helped ensure the quality of the papers. We hope this special 
issue will stimulate the continuing efforts on research and technology development of biomass-based materials and technologies for energy-related applications.

Jingxin Wang

Xinfeng Xie

David B. DeVallance

Jianchun Jiang

Lixin Huang

Levente Dénes 

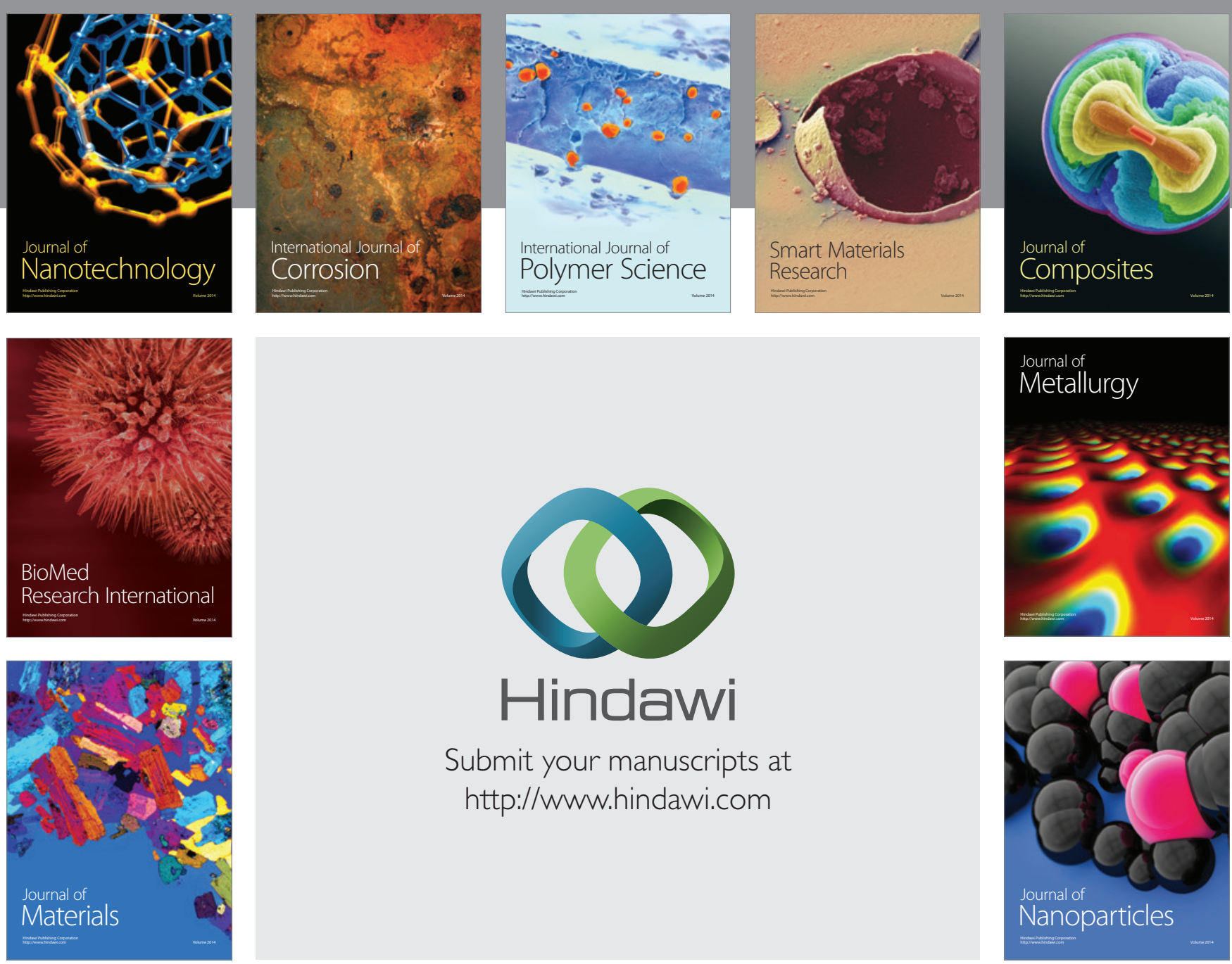

Submit your manuscripts at http://www.hindawi.com
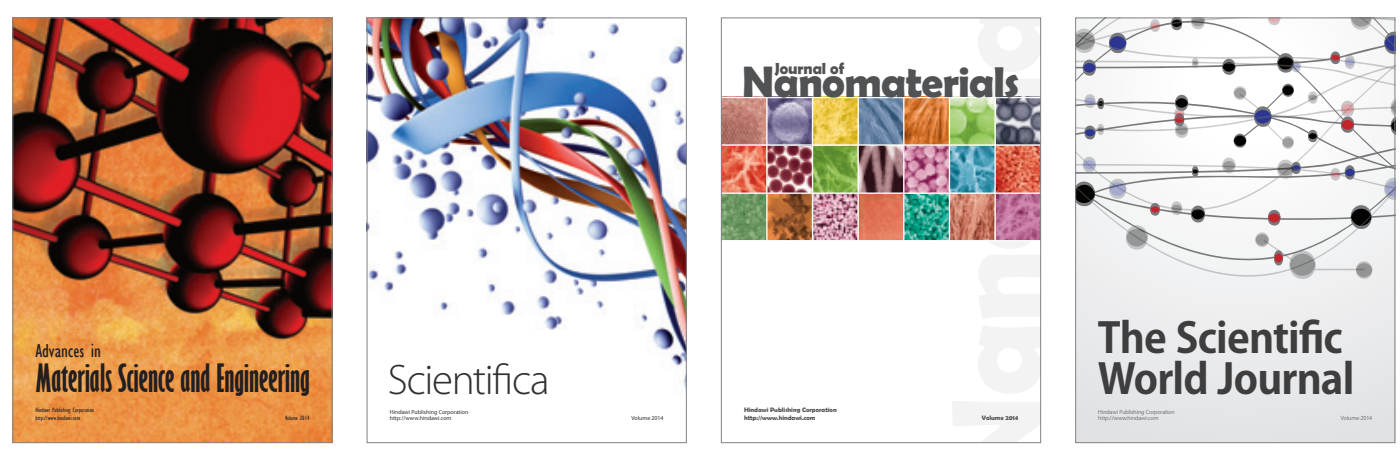

\section{The Scientific World Journal}
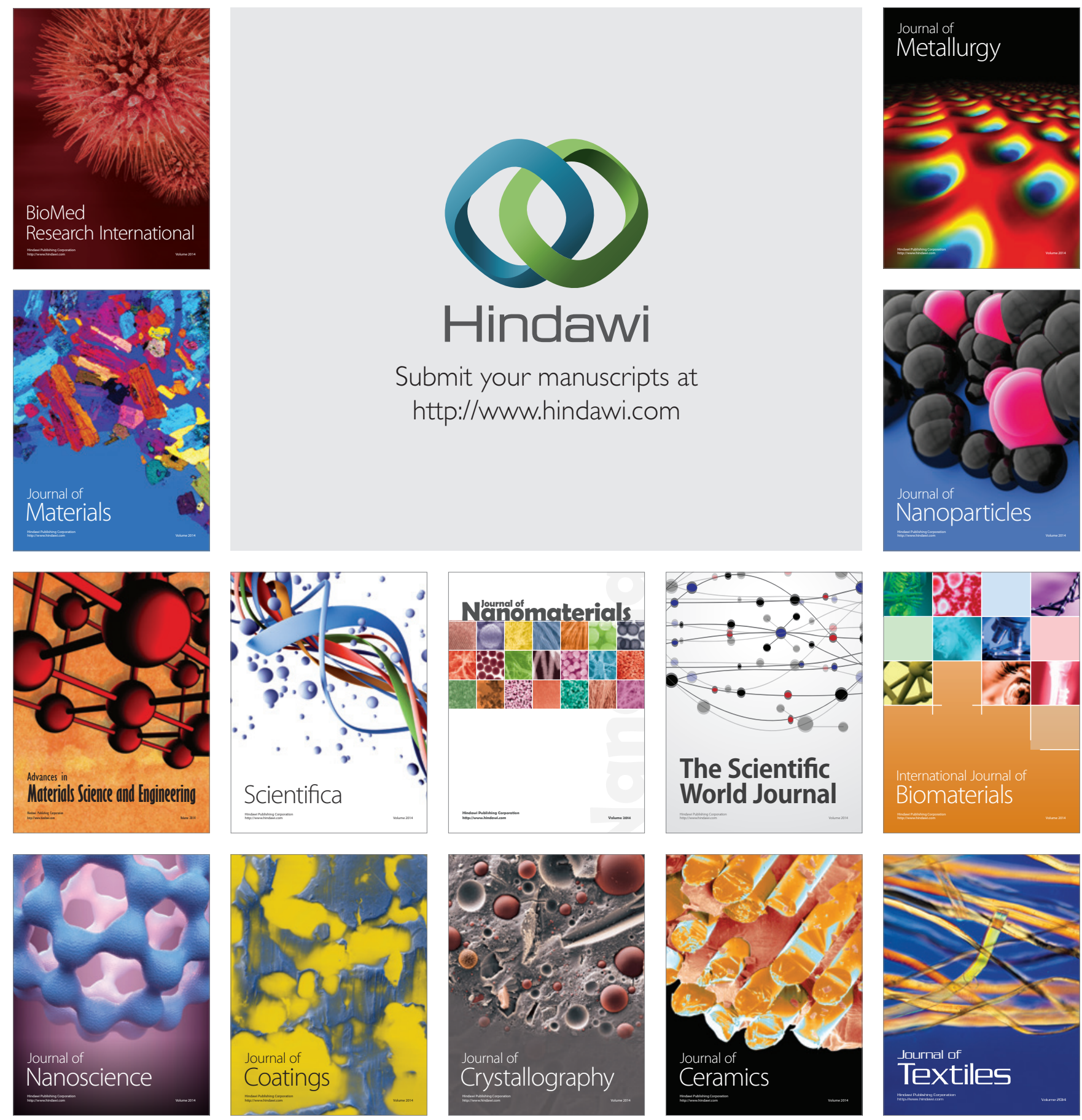